\title{
Bark Beetle Conversion of a Plant Compound to a Sex-Specific Inhibitor of Pheromone Attraction
}

John A. Byers 


\section{Bark Beetle Conversion of a Plant Compound to a Sex-Specific Inhibitor of Pheromone Attraction}

Abstract. Both sexes of the bark beetle Dendroctonus brevicomis convert the $(+)$ and (-) enantiomers of the tree terpene $\alpha$-pinene to the corresponding enantiomers of trans-verbenol at about equal rates. (-)-trans-Verbenol inhibited the response of females, but not of males, to a mixture of attractive pheromone components. Since the female initiates the attack on a pine tree, (-)-trans-verbenol may play a role in reducing intraspecific competition for breeding areas.

Many species of bark beetle have pheromones that incite individuals to collect in a mass attack on a tree for the purposes of finding mates and locating suitable breeding areas; this often results in the death of the tree. Some species use an olfactory mechanism to avoid interspecific competition for hosts $(1-3)$ as well as to reduce intraspecific competition (4). The Western pine beetle Dendroctonus brevicomis LeConte is a destructive species infesting ponderosa pine, the predominant forest tree in California. Male and female beetles are equally attracted to a mixture of three pheromone components: exo-brevicomin, produced by females; frontalin, produced by males; and myrcene, derived from tree resin (5).

Racemic $\alpha$-pinene, a major monoterpene of the pine tree (6), is apparently hydroxylated by $D$. brevicomis females to form trans-verbenol (7), which accumulates in the hindguts at the beginning of colonization $(2,8)$. However, despite several attempts, no behavioral or ecological function has been established for trans-verbenol $(9,10)$. I have found that the (-) enantiomer of trans-verbenol acts to inhibit the attraction of females, but not of males, to pheromone components. Since the female begins the attack on a tree, this inhibitory response appears to play a role in regulating the density of colonization and intraspecific competition. Renwick et al. (II) reported that the cohabiting bark beetle Ips paraconfusus converted the (+) enantio- mer of $\alpha$-pinene to trans-verbenol and converted the ( - ) enantiomer to an attractive pheromone component, cis-verbenol. In contrast, I found that both sexes of $D$. brevicomis use both enantiomers of $\alpha$-pinene to synthesize the corresponding enantiomers of trans-verbenol (12) and synthesize only trace amounts, if any, of cis-verbenol.

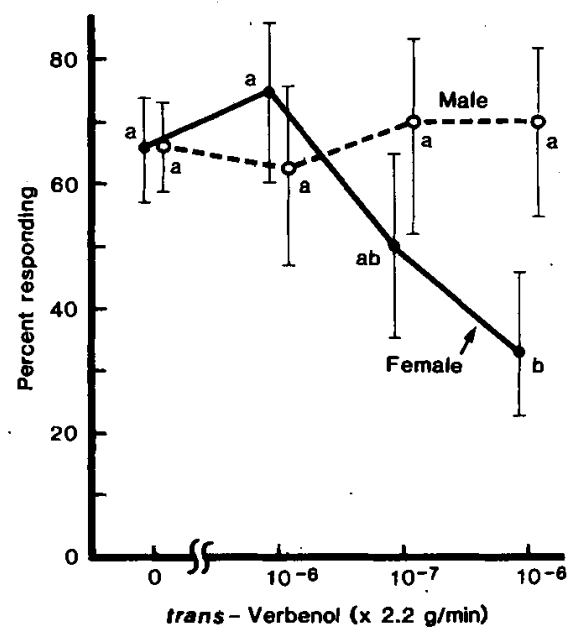

Fig. 1. Effect of increasing release rates of (-)-trans-verbenol on the attraction response of walking male and female Dendrostonus brevicomis to a 1:1:1 mixture of pheromone components exo-brevicomin (>95 percent), frontalin ( $>95$ percent), and myrcene ( $>99$ percent) (Chemical Samples Company), each released at $2.2 \times 10^{-9} \mathrm{~g} / \mathrm{min}$ (18 October 1976). Points with the same letter were not significantly different $\left(\alpha=.01, \chi^{2}\right)$. The brackets represent 95 percent confidence limits for proportions.
Adult $D$. brevicomis were reared in the laboratory from the bark of larva-infested ponderosa pine trees (Sierra National Forest, California) and were used in experiments shortly after they emerged. The beetles were exposed for 18 hours to vapors of either the $(+)$ or $(-)$ enantiomer of $\alpha$-pinene (Aldrich), purified $>99.8$ percent by gas-liquid chromatography (GLC). The quantities of transverbenol, verbenone, and myrtenol in the hindguts were determined by GLC (13) (Table 1). The amounts of transverbenol produced from each enantiomer of $\alpha$-pinene were similar in males and females, as were the amounts of myrtenol (Table 1). Females did not produce verbenone in detectable levels, whereas males produced significant amounts after being held in a jar for 18 hours at room temperature, whether or not they were exposed to $\alpha$-pinene vapors. A beetle of either sex contained no more than $0.5 \times 10^{-7} \mathrm{~g}$ of cis-verbenol (quantified by GLC) after exposure to (-)- $\alpha$-pinene, not significantly different from the amount in unexposed beetlesbut exposure to $(+)$ - $\alpha$-pinene resulted in about $5 \times 10^{-7} \mathrm{~g}$ of a compound having the retention time of cis-verbenol.

Samples of trans-verbenol were collected in ethanol from the gut extracts (diethyl ether) by condensation on glass beads from the GLC effiuent (14). The concentrations were quantified by GLC, and the optical rotations ( \pm 5 percent) were obtained with an electrobalancing polarimeter (Autopol III). A comparison of the specific rotations of the $(+)$ - and $(-)$ - $\alpha$-pinene with the specific rotations of $(+)$ - and (-)-trans-verbenol produced in males $\left([\alpha]_{\mathrm{D}}{ }^{22}=+105.2^{\circ},-89.5^{\circ}\right)$ and females $\left([\alpha]_{D}{ }^{22}=+104.9^{\circ},-91.5^{\circ}\right)$ indicated that both sexes converted both enantiomers, but each only to the corresponding enantiomer of trans-verbenol (I5). The GLC-purified samples of transverbenol were then subjected to analysis by gas chromatography-mass spectrometry (V.G. Micromass 7070F mass spectrometer with computerized data system) on a 40-m capillary column (SCOT; $\mathrm{OV} 101$ ) at $115^{\circ} \mathrm{C}$. The trans-verbenol extracted from males and females had mass spectra identical to the mass spectrum of a standard purified $>99.8$ percent by GLC (Glidden Organics) (16).

Walking beetles were tested with a laboratory olfactometer for their response to a mixture of pheromone components containing increasing concentrations of trans-verbenol, $[\alpha]_{D}{ }^{22}=-131^{\circ}$ (Glidden Organics) (3). Only the response of females to pheromone components was inhibited at the highest release 
rate of trans-verbenol (Fig. 1). Females were further tested to determine whether both enantiomers could inhibit attraction. The predominantly (+)- or predominantly (-)-trans-verbenol obtained from the females exposed to the respective enantiomers of $\alpha$-pinene was released at $6.6 \times 10^{-7} \mathrm{~g} / \mathrm{min}$ in the olfactometer with a mixture of the attractive pheromone components (each released at $\left.1.2 \times 10^{-8} \mathrm{~g} / \mathrm{min}\right)$. A female response of 68.3 percent $(N=180)$ to the pheromone mixture was unaffected by $(+)$ trans-verbenol (68.9 percent, $N=90$ ), but attraction was reduced by (-)-transverbenol (52.2 percent, $N=90$, $\left.P<.05, \chi^{2}\right)$.

A field test, on 5 to 12 August 1980, in which five pairs of traps were placed in various areas of the Sierra National Forest (elevation $1000 \mathrm{~m}$ ), was designed to determine whether (-)-trans-verbenol could influence the sex ratio of beetles landing and entering beetle-sized holes in a paper carton containing pheromone components. The carton $(19 \mathrm{~cm}$ in diameter and $21.5 \mathrm{~cm}$ high) had 48 holes (2.5 $\mathrm{mm}$ ) evenly spaced around the sides and an adhesive coating (Stickem Special) (2) on the inside floor. Glass vials containing the three pheromone components, each released at about $2 \mathrm{mg} / \mathrm{day}$, and two vials containing (-)-trans-verbenol, released at about $0.5 \mathrm{mg} / \mathrm{day}$ (2), were placed inside the carton. A $13-\mathrm{cm}^{2}$ wire screen (6-mm mesh) coated with adhesive was placed immediately below the carton. Both carton and screen were attached to a wooden stake $1.5 \mathrm{~m}$ above ground and $8 \mathrm{~m}$ away from the control traps, which did not contain trans-verbenol. The sticky screen on the control traps caught 95 males and 93 females. This sex ratio was similar to that of beetles caught on screens below cartons containing transverbenol (61 males and 64 females), an indication that trans-verbenol did not significantly affect the long-distance attraction to pheromone. Inside the control cartons there were 75 males and 56 females, and this sex ratio was not significantly different from the ratio of those caught on the screens below $(P>.05$, $\left.\chi^{2}\right)$. However, the sex ratio of beetles caught inside the cartons releasing transverbenol (93 males and 21 females) was significantly different from the sex ratio of beetles on the screens below and from the ratio of beetles caught on the inside of the control cartons $\left(P<.001, \chi^{2}\right)$. This result supports the laboratory finding that trans-verbenol inhibits the response of females, since females appeared less likely than males either to enter a hole from which trans-verbenol was being released or to remain on the outside of the cartons.

Each of the two enantiomers of $\alpha$ pinene is converted by both sexes of $D$. brevicomis to the comparable enantiomer of trans-verbenol, but only the (-) enantiomers of precursor and product appear to be involved in the behavioral ecology of the beetle. In the cohabiting bark beetle $I$. paraconfusus the biosynthetic system which produces cis- and trans-verbenol from $\alpha$-pinene seems fundamentally different from that in $D$. brevicomis $(11,17)$. Both cis- and transverbenol have been produced in culture by a bacterium isolated from $I$. paraconfusus (18), but when these beetles were fed an antibiotic at concentrations that abolished synthesis of two other pheromone components, ipsenol and ipsdienol, there was no apparent effect on verbenol production (19). In contrast, $D$. brevicomis produced no cis-verbenol or only trace amounts from (-)- $\alpha$-pinene, and only a small peak, about 4 percent relative to trans-verbenol, was detected after exposure to $(+)$ - $\alpha$-pinene. This may be because $D$. brevicomis appears to use $(+)$-cis-verbenol for detecting and avoiding areas of the tree infested with $I$. paraconfusus in order to reduce competition $(2,3)$. Both species probably use $(-)-\alpha$-pinene to make both attractive and inhibitory pheromone components because this enantiomer predominates in ponderosa pine resin (6).

Table 1. Amounts of trans-verbenol, verbenone, and myrtenol in hindguts of male and female $D$. brevicomis when exposed or not exposed to vapors of each enantiomer of $\alpha$-pinene. There were no significant differences between males and females in the amount of trans-verbenol or myrtenol produced from each enantiomer of $\alpha$-pinene or in the amounts of trans-verbenol or myrtenol produced from each enantiomer by either sex $(P>.05)$. Significantly more transverbenol and myrtenol were produced in males and females exposed to enantiomers of $\alpha$-pinene than in controls not exposed to vapor for 18 hours $(P<.001)$.

\begin{tabular}{|c|c|c|c|}
\hline \multirow{2}{*}{ Treatment } & \multicolumn{3}{|c|}{ Production per beetle $\left(\times 10^{-7} \mathrm{~g}\right)$} \\
\hline & trans-Verbenol & Verbenone & Myrtenol \\
\hline \multicolumn{4}{|c|}{ Male } \\
\hline$(+)-\alpha$-pinene ${ }^{*}$ & $58.5 \pm 9.8$ & $6.4 \pm 1.5$ & $20.5 \pm 2.9$ \\
\hline$(+)$ - $\alpha$-pinene $\dagger$ & $135.3 \pm 4.8$ & $13.3 \pm 2.7$ & $44.9 \pm 4.8$ \\
\hline$(-)-\alpha$-pinene* & $74.6 \pm 10.8$ & $12.4 \pm 0.6$ & $10.7 \pm 1.5$ \\
\hline$(-)$ - $\alpha$-pinene $\dagger$ & $218.1 \pm 20.6$ & $22.9 \pm 5.1$ & $28.6 \pm 3.4$ \\
\hline None & $0.5 \pm 0.1$ & $6.2 \pm 2.4$ & $0.2 \pm 0.1$ \\
\hline No vapor (18 hours) $\ddagger$ & $3.0 \pm 0.5 \S$ & $27.9 \pm 3.6$ & $0.8 \pm 0.3$ \\
\hline \multicolumn{4}{|c|}{ Female } \\
\hline$(+)-\alpha$-pinene ${ }^{*}$ & $65.9 \pm 13.9$ & $<0.1 \|$ & $29.0 \pm 6.4$ \\
\hline$(+)$ - $\alpha$-pinene $\dagger$ & $139.9 \pm 16.7$ & $<0.1 "$ & $45.2 \pm 5.9$ \\
\hline$(-)-\alpha$-pinene $*$ & $89.7 \pm 9.4$ & $<0.1$ & $17.7 \pm 1.4$ \\
\hline$(-)-\alpha$-pinene $\dagger$ & $208.8 \pm 28.9$ & $<0.1$ & $37.6 \pm 5.5$ \\
\hline None $\neq$ & $0.7 \pm 0.1$ & $<0.1$ & $0.1 \pm 0.1$ \\
\hline No vapor (18 hours) $\ddagger$ & $6.6 \pm 1.3 \S$ & $<0.1$ & $0.6 \pm 0.1$ \\
\hline
\end{tabular}

* Two groups of 100 beetles of each sex were exposed to each enantiomer of $\alpha$-pinene $\left([\alpha]_{\mathrm{D}}^{22}=+45.8^{\circ}\right.$; $\left.[\alpha]_{0}^{22}=-41.6^{\circ}\right)$ at $4.9 \pm 0.6 \times 10^{-6} \mathrm{~g}$ per milliliter of air for 18 hours on 15 December $1977(/ 3)$. $\neq$ Six groups of 20 beetles of each sex were exposed to each enantiomer of $\alpha$-pinene at $7.8 \pm 0.8 \times 10^{-6} \mathrm{~g}$ per milliliter of air for 18 hours (two groups each on 13 October and 16 November 1977 and on 3 May 1980). $\quad$ Two groups of 15 beetles of each sex were not exposed to vapors at any time, or for 18 hours, on each of the three dates above. \$Both sexes contained significantly more Irans-verbenol and males more ver jar for 18 hours at room temperature than corresponding beetles th emerged and were immediately refrigerated $\left(3^{\circ} \mathrm{C}\right)$ for 18 hours until extraction $(P<.001)$. \| Females did not produce detectable amounts of verbenone.
The largest quantities of trans-verbenol are found in female $D$. brevicomis quantities are comparable to the duced by the female when gallery construction under bark is begun $(2,20)$. The males to avoid areas with high densities boring females and would thus reguamount of trans-verbenol in the hindguts of both males and females diminishes attractive pheromone components decline more rapidly (20). The change in the ratio of these substances may inditack sequence that the tree is fully colonized. It would be advantageous for benol-as they apparently were notsince they seek mates and sources of food and protection. However, it would tive success by complementing the female's production of trans-verbenol, since his progeny would be as much that of his mate.

The present study provides a method of trans-verbenol for use in behavioral experiments with other bark beetles such as the Southern pine beetle, mountain pine beetle, and Douglas-fir beetle, which use the compound as an aggrega- 
tion signal (2l). The field trap design illustrates a means of determining the behavioral activity of repellent or inhibitory pheromones under conditions similar to those in nature. trans-Verbenol, alone or in combination with other inhibitors, such as verbenone $(10,22)$, ipsdienol (14), or cis-verbenol (3), may be useful in protecting trees from attack by D. brevicomis.

JOHN A. BYERs* Department of Entomological Sciences, University of California,

Berkeley 94720

\section{References and Notes}

1. M. C. Birch and D. L. Wood, J. Chem. Ecol. 1, 101 (1975).

2. J. A. Byers and D. L. Wood, ibid. 6, 149 (1980).

3. , ibid. 7, 9 (1981)

4. J. A. Rudinsky, Science 166, 884 (1969) and R. R. Michael, ibid. 175, 1386 (1972); G. B Pitman and J. P. Vité, Environ. Entomol. 3, 886 (1974); J. A. Byers, J. Chem. Ecol. 9, 129(1983). (1974); J. A. Byers, J. Chem. Ecol. 9 , 129 (1983).
R. M. Silverstein, R. G. Brownlee, T. E. Bellas D. L. Wood, L. E. Browne, Science 159, 889 (1968); G. W. Kinzer, A. F. Fentiman, Jr., T. F Page, Jr., R. L. Foltz, J. P. Vité, G. B. Pitman Nature (London) 22, 475 (1969); D. L. Wood et al., Science 192, 896 (1976).

6. Both $(+)$ and $(-)$ enantiomers of $\alpha$-pinene occur in ponderosa pine resin, but the $(-)$ enantiomer predominates in California [ $E$ Zavarin and $F$. predominates in California [E. Zavarin and F.

7. P. R. Hughes, Z. Angew. Entomol. 73, 294 P. R. H

8. J. A. A. Renwick, Contrib. Boyce Thompson Inst. 23, 355 (1967).

9. —, ibid. 24, 337 (1970).

10. and J. P. Vité, ibid p. 283.

11. J. A. A. Renwick, P. R. Hughes, I. S. Krull, Science 191, $199(1976)$ [in figure $2(+)$-trans verbenol and $(+)$-myrtenol should have been represented by the $(1 R, 4 S, 5 R)$ configuration instead of the $(1 S, 4 R, S S)$ model shown].

12. Hydroxylation of $(I R)-(+)$ - and $(I S)-(-)-\alpha-p i-$ nene enantiomers (non-superimposable mirro images) to $(1 R)-(+)$ - and $(1 S)-(-)$-trans-verbeno enantiomers in $D$. brevicomis:

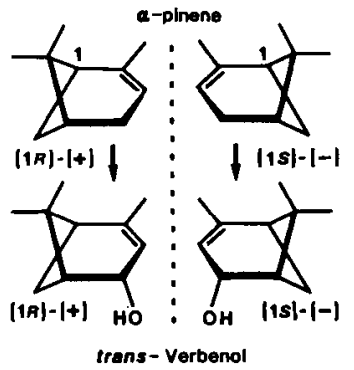

13. J. A. Byers, D. L. Wood, L. E. Browne, R. H Fish, B. Piatek, L. B. Hendry, J. Insect Physiol. 25, 477 (1979)

14. J. A. Byers, J. Chem. Ecol. 8, 363 (1982).

15. The same proportions of $(+)$ and $(-)$ enantiomers found in the samples of $(+)$ and $(-)-\alpha-$ mers found in the samples of $(+)$ and $(-)-\alpha-$ pinene were found in the beetle-produced $(+)$ and (-)-trans-verbenol, as judged by the highest reported rotations for $(-)$-trans-verbenol
$\left([\alpha]_{D}^{22}=-129.6^{\circ}\right)$ and $(+)-\alpha$-pinene $\left([\alpha]_{D}^{22}=\right.$ $\left([\alpha]_{D}{ }^{22}=-129\right.$.
$\left.+52.4^{\circ}\right)(I I)$.

16. The trans-verbenol had a molecular weight of 152 and mass spectra (mass-to-charge ratio) in decreasing magnitude from a base peak of 109 : 94 (59 percent of base peak) $91,81,119,95,69$ $83,137,92,84,41,67,107,79$, and 134 which 83 , $137,92,84,41,61$, were in agreement with spectra of other samples of trans-verbenol obtained by G. Odham, Uniof Göteborg. Sweden. However, the spectra were not in agreement with those obtained by $\mathrm{J}$. A. A. Renwick (8), who reported a base peak of 119 and major fragments of 91 and 134 for transverbenol obtained from $D$. brevicamis

17. J. A. Byers, Insect Biochem. 11, 563 (1981)

18. J M Brand, J. W. Bracke A. J Markovetz, D. L. Wood, L. E. Browne, Nature (London) 254, 136 (1975).
19. J. A. Byers and D. L. Wood, Science 213, 763 20. (1981)

20. $\frac{}{\text { tion. }}$, J. Craig, L. B. Hendry, in prepara-

21. J. A. A. Renwick and J. P. Vité, Nature (London) 224, 1222 (1969); G. B. Pitman and J. P. Vité, Can. Entomol. 101, 143 (1969); J. A. Rudinsky and R. R. Michael, in (2)

22. W. D. Bedard, P. E. Tilden, K. Q. Lindahl, Jr., D. L. Wood, P. A. Rauch, J. Chem. Ecol. 6, 997 (1980)

23. I thank D. L. 'Wood, G. O. Poinar, Jr., C.
Löfstedt, $J$. Löfqvist, and F. Schlyter for reviews; W. F. Haddon and E. Zavarin for assistance in the chemical analyses; and W. D. Be dard and P. E. Tilden for use of their research facilities. Supported in part by grants from the Rockefeller Foundation and the U.S. Forest Service, and by Regional Research Project grant W-I 10, SEA/USDA (to D. L. Wood).

- Present address: Department of Animal Ecology, University of Lund, S-22362 Lund, Sweden.

3 August 1982; revised 2 November 1982 